\title{
LOS PROBLEMAS DE EFECTIVIDAD EN EL PROCESO DE EVALUACION DE IMPACTO AMBIENTAL DE COSTA RICA Y SUS CONSECUENCIAS PARA LA GESTIÓN DEL GEÓLOGO
}

\author{
THE PROBLEMS OF EFFECTIVENESS IN THE ENVIRONMENTAL \\ IMPACT ASSESSMENT IN COSTA RICA AND ITS CONSEQUENCES FOR \\ GEOLOGICAL MANAGEMENT
}

\author{
Allan Astorga-Gättgens \\ Escuela Centroamericana de Geología, UCR \\ Consultor en Gestión Ambiental Integral, Evaluación Ambiental y \\ Ordenamiento Territorial. Apto. 480 2070, San José, Costa Rica \\ a.astorga.g@gmail.com
}

(Recibido: 17/09/2015; aceptado: 11/12/2015)

\begin{abstract}
This document presents the results of a process of technical review of nearly 60 records of environmental impact assessment (EIA) carried out in recent years as part of the course of Environmental Geology I. The main problems detected and most common indicated in most records. This concludes that there are serious problems in implementing existing procedures and current technical tools, by both environmental consultants and the "Secretaría Técnica Nacional Ambiental" (SETENA). To this situation, the environmental management consultant geologist is not the exception, reason why it is necessary that the above mentioned professionals are aware of the severe legal consequences involved. Finally, this paper proposes five key solutions to correct and improve the serious efficiency problem of the EIA system, which has brought the country to a condition of unsustainable development.

Keywords: Environmental Impact Assessment (EIA), Environmental Consultant, legislation, procedures, efficiency, environmental management.
\end{abstract}

\footnotetext{
RESUMEN: Se presentan los resultados de un proceso de revisión técnica de cerca de 60 expedientes de evaluación de impacto ambiental (EIA) realizado como parte del curso de Geología Ambiental I. Se indican los principales problemas detectados, comunes a la mayoría de los expedientes. Se concluye que existen serios problemas de aplicación de los procedimientos e instrumentos técnicos vigentes, tanto por parte de los consultores ambientales como de la misma Secretaría Técnica Nacional Ambiental (SETENA). A esta situación no escapa la gestión ambiental del geólogo consultor, razón por la cual, se hace necesario que dichos profesionales conozcan las serias consecuencias legales que ello implica. Finalmente, se plantean cinco soluciones clave para corregir y mejorar el grave problema de eficiencia que tiene el sistema de EIA, el cual ha llevado al país a una condición de desarrollo no sustentable.

Palabras clave: Evaluación de Impacto Ambiental (EIA), Consultor Ambiental, legislación, procedimientos, eficiencia, gestión ambiental.
} 


\section{INTRODUCCIÓN}

La Evaluación de Impacto Ambiental (EIA) es un instrumento de gestión ambiental pública que se aplica desde hace más de 45 años en muchos países del mundo (ver Canter, 1995). Se define como "la identificación y valoración de los impactos (efectos) potenciales de proyectos, planes, programas o acciones normativas relativos a los componentes físico - químicos, bióticos, culturales y socioeconómicos del entorno" (in ibíd., 1995). En Costa Rica se aplica, de forma particular, desde el año 1982 cuando el Código de Minería estableció el requisito de que todos los proyectos mineros, debían cumplir con el requisito de elaborar un Estudio de Impacto Ambiental de previo a su ejecución.

La EIA se aplica de forma más sistemática en Costa Rica desde el año 1995 cuando se promulgó la Ley Orgánica del Ambiente (Ley 7554). Esta Ley, creó la Secretaría Técnica Nacional Ambiental (SETENA) como un ente de desconcentración máxima, adscrita al Ministerio de Ambiente y Energía (MINAE) con la tarea de administrar todo lo relacionado con la EIA, y decidir sobre la viabilidad ambiental de los proyectos, obras o actividades de desarrollo.

Como parte del curso de Geología Ambiental I que el autor imparte en la Escuela Centroamericana de Geología de la Universidad de Costa Rica, se desarrolla el tema de la EIA. En dicho curso, durante la última década, se ha procedido a realizar una investigación dirigida que implica la revisión de diferentes expedientes de EIA de proyectos que se han tramitado en la SETENA. Se han revisado, diversos tipos de proyectos (urbanísticos, mineros, industriales, turísticos, hidroeléctricos, etc.) a lo largo de todo su ciclo de trámite hasta que obtienen la viabilidad ambiental.

En el presente documento técnico, se presentan las principales observaciones técnicas derivadas de ese trabajo de investigación realizado durante la última década y que implica la revisión de cerca de 60 expedientes administrativos de EIA.

La metodología aplicada en la revisión ha comprendido el cotejo del procedimiento aplicado en el expediente, respecto a la legislación vigente en materia de EIA, tanto en el Reglamento General de Procedimientos de EIA, como en el Manual Técnico de EIA vigente en Costa Rica (ver Cuadro 1).

El objetivo de la presente nota técnica es aportar los resultados obtenidos de la investigación a fin de que sirva de registro para promover una discusión abierta sobre el tema de EIA en Costa Rica, y así, facilitar la toma de acciones correctivas que lleven a mejorar de forma sistemática ese importante procedimiento de gestión ambiental administrativa.

Es importante señalar que en razón de que se trata de proyectos, obras y actividades de inversión económica, que en la mayoría ya fueron construidos y se encuentran en operación, no se hace aquí referencia específica al nombre o número de expediente administrativo. Tampoco se hace referencia alguna al nombre de los consultores ambientales autores de los estudios técnicos, ni de los evaluadores ambientales que tomaron parte de la revisión en la SETENA. Esto, en razón de que no fue parte del objetivo de la investigación, la cual se centra en una valoración genérica del proceso de EIA tomando como base unos expedientes de "muestra", sin entrar en otro tipo de detalles.

\section{RESULTADOS PRINCIPALES}

Los resultados obtenidos son muchos e infortunadamente, no son buenos. No obstante, se considera muy importante poder reportarlos, siempre con la sana intención de que se tomen en cuenta y sirvan de base para realizar un cambio. A fin de aprovechar el máximo el espacio y de no entrar en polémicas, respecto al nombre del proyecto, los desarrolladores o el equipo consultor responsable de la EIA, no se menciona ninguna de esta información. Vamos directo a los hallazgos principales.

Los resultados de la revisión de diversos expedientes ambientales durante los últimos 10 años, muestran una serie importante de deficiencias en el proceso de EIA. En lo que sigue se enumeran y explican brevemente, los principales resultados.

1. Incumplimiento de plazos: en el casi 100 $\%$ de los casos, la SETENA no cumple los plazos 
que le establece la legislación. En algunos casos, esos plazos se exceden en hasta 5 veces el plazo original.

2. Los formularios D1 no son llenados correctamente: los consultores, por lo general, ponen el caso más simple, para forzar que el puntaje resulte bajo.

3. Los consultores responsables, en muchos casos, no asumen la verdadera responsabilidad de efectuar una eficiente EIA, Juegan un papel de "defensor" a ultranza de los intereses del desarrollador que los ha contratado.

4. Los estudios técnicos complementarios, de ingeniería, geología y arqueología, así como los de biología o de impacto vial, por lo general, no se integran de forma efectiva al diseño del proyecto. Se realizan más bien como un requisito a cumplir.

5. Dichos estudios técnicos, en muchos casos, son hechos con gran generalidad, en una escala más amplia de lo solicitada, y sin cumplir de forma estricta los procedimientos técnicos.

6. Las medidas ambientales incluidas como complemento al formulario D1 y al Pronóstico Plan de Gestión Ambiental (PPGA), por lo general, son "caprichosas", en la medida de que se plantean para solucionar una situación específica, pero sin cumplir un estándar básico de referencia, lo que hace que existan muchas asimetrías de gestión ambiental para proyectos similares.

7. En muchos de los instrumentos técnicos de EIA, se nota la intención de muchos consultores de "simplificar" la situación, a fin de justificar el diseño del proyecto, con lo cual, se induce a error a la entidad que revisa los documentos, la cual, en muchos casos, acepta la información sin ningún cuestionamiento.

8. Tanto en D1, como en PPGA o en Estudios de Impacto Ambiental (EsIA), es clara la ausencia de una verdadera evaluación ambiental, en la medida de que se valoren realmente los impactos estratégicos del proyecto, y cómo las medidas deben ser integradas en el diseño del proyecto, para mejorarlo. El proceso que se sigue, tanto por el equipo consultor, como por la misma SETENA, es mecánico, sin que se cumpla el objetivo fundamental de la EIA.

9. En muchos casos, los impactos ambientales no son correctamente identificados, ni valorados según la metodología estándar establecida en la normativa. Este aspecto es particularmente grave, en la medida de que impactos importantes son eliminados o subestimados, sin que nadie justifique por qué fueron excluidos.

10. En la valoración de los impactos, no se da la objetividad necesaria, es muy común detectar que el consultor ambiental "fuerza" los valores para que los resultados den bajos puntajes, es decir, que los impactos ambientales resulten bajos.

11. Con los años, es cada vez más frecuente el proceso de segmentación de la EIA, de forma tal que un proyecto grande, se separa, bajo diversos "pretextos" en una serie de pequeños componentes que hacen que se pierda la verdadera visión del conjunto del proyecto y por tanto, la EIA pierde totalmente la eficacia.

12. La SETENA no utiliza en la totalidad los instrumentos y procedimientos técnicos de que dispone, como por ejemplo, la posibilidad de solicitar Planes Ambientales de Desarrollo, para proyectos grandes (inmobiliarios, turísticos, urbanísticos), que se desarrollan en terrenos privados, sin plan regulador que los norme. Esto lleva a que se cometan errores fundamentales como aprobación de propuestas de diseños de sitio inconsistentes con temas como las amenazas naturales o la vulnerabilidad acuífera.

13. Existe una seria deficiencia en los procesos de control y seguimiento en control ambiental de las actividades. Los informes ambientales de los responsables (regentes) ambientales no son revisados sistemáticamente por la SETENA. El departamento de Auditoría y Seguimiento Ambiental es muy pequeño y ocupa casi todo su tiempo en el tema de las garantías ambientales. No se realizan procesos de auditorías ambientales de cumplimiento, ni la calificación ambiental de las actividades según establece el reglamento.

La falta de eficiencia y eficacia de los consultores ambientales en la preparación de los instrumentos de EIA, se traslada a la SETENA, ya que esta entidad acepta la información tal y como se le presenta, sin generar dudas de fondo. Esto, a pesar de que en muchos casos la situación es muy evidente. No obstante, eso no evita que la SETENA solicite información complementaria, pero en muchos casos poco significativa para el proceso de 
Cuadro 1

Instrumentos y procedimientos técnicos de EIA, vigentes en Costa Rica.

\begin{tabular}{|c|c|c|}
\hline Nombre & Instrumento & Observación \\
\hline \multicolumn{3}{|l|}{ Decretos ejecutivos } \\
\hline $\begin{array}{c}\text { Reglamento General sobre los Proced- } \\
\text { imientos de Evaluación de Impacto } \\
\text { Ambiental (EIA) }\end{array}$ & $\begin{array}{l}\text { Decreto Ejecutivo 31849-(MINAE- } \\
\text { SALUD-MOPT-MAG-MEIC, 2004) }\end{array}$ & $\begin{array}{c}\text { Marco general, técnico y legal del } \\
\text { proceso de EIA. Además de sus modifi- } \\
\text { caciones }\end{array}$ \\
\hline $\begin{array}{l}\text { Instructivo de llenado Formulario D2 y } \\
\text { Código de Buenas Prácticas Ambientales }\end{array}$ & $\begin{array}{c}\text { Decreto Ejecutivo 32079-(MINAE, } \\
\text { 2004) }\end{array}$ & $\begin{array}{c}\text { Formulario D2 y guía de llenado y el } \\
\text { Código de Buenas Prácticas Ambientales }\end{array}$ \\
\hline Instructivo de llenado Formulario D1 & $\begin{array}{c}\text { Decreto Ejecutivo } 32712-(\mathrm{MINAE}, \\
\text { 2005a) }\end{array}$ & $\begin{array}{l}\text { Formulario D1 y guía de llenado. } \\
\text { Además de sus modificaciones }\end{array}$ \\
\hline Creación Comisión Mixta & $\begin{array}{l}\text { Decreto Ejecutivo 32631-(MINAE, } \\
\text { 2005b) }\end{array}$ & $\begin{array}{c}\text { Comisión Mixta de apoyo a la SETENA. } \\
\text { Además de sus modificaciones }\end{array}$ \\
\hline $\begin{array}{l}\text { Guía para la elaboración de instrumentos } \\
\text { de EIA }\end{array}$ & $\begin{array}{c}\text { Decreto Ejecutivo 32966-(MINAE, } \\
\text { 2006a) }\end{array}$ & $\begin{array}{l}\text { Guía General de EIA y procedimiento de } \\
\text { valoración de impactos ambientales }\end{array}$ \\
\hline Protocolo Planes Reguladores & $\begin{array}{l}\text { Decreto Ejecutivo 32967-(MINAE, } \\
\text { 2006b) }\end{array}$ & $\begin{array}{l}\text { Procedimiento para introducir la variable } \\
\text { ambiental en los planes reguladores y } \\
\text { planificación de uso del suelo }\end{array}$ \\
\hline $\begin{array}{l}\text { Manual de Instrumentos Técnicos para } \\
\text { el Proceso de Evaluación de Impacto } \\
\text { Ambiental (Manual de EIA) -PARTE V }\end{array}$ & $\begin{array}{l}\text { Decreto Ejecutivo 33959-(MINAE, } \\
\text { 2007) }\end{array}$ & $\begin{array}{l}\text { Procedimiento técnico y ambiental para } \\
\text { los movimientos de tierras }\end{array}$ \\
\hline $\begin{array}{l}\text { Reglamento de fijación de tarifas de } \\
\text { servicios brindados por la Secretaría } \\
\text { Técnica Nacional Ambiental }\end{array}$ & $\begin{array}{c}\text { Decreto Ejecutivo } \\
\text { 34536-(MINAE,2008 a) }\end{array}$ & $\begin{array}{c}\text { Tarifario de costos para EIA que aplica } \\
\text { la SETENA }\end{array}$ \\
\hline Guías Ambientales & $\begin{array}{l}\text { Decreto Ejecutivo 34522-(MINAE, } \\
\text { 2008b) }\end{array}$ & $\begin{array}{l}\text { Procedimiento para formalización de } \\
\text { guías de buenas prácticas ambientales }\end{array}$ \\
\hline $\begin{array}{c}\text { Reglamento de Organización de la } \\
\text { Estructura Interna de Funcionamiento de } \\
\text { la SETENA }\end{array}$ & $\begin{array}{l}\text { Decreto Ejecutivo } \\
\text { 36815-(MINAET,2011) }\end{array}$ & $\begin{array}{l}\text { Organización y funcionamiento de la } \\
\text { SETENA }\end{array}$ \\
\hline \multicolumn{3}{|l|}{ Resoluciones de la SETENA } \\
\hline $\begin{array}{l}\text { Trámite Actividades, Obras o Proyectos } \\
\text { MUY BAJO IMPACTO AMBIENTAL }\end{array}$ & 2370-2004-SETENA & $\begin{array}{l}\text { Lista de actividades de muy bajo im- } \\
\text { pacto ambiental }\end{array}$ \\
\hline Georeferención de Proyectos & 2654-2008-SETENA & $\begin{array}{c}\text { Procedimiento para la georeferenciación } \\
\text { de áreas de proyecto }\end{array}$ \\
\hline Proyectos de Muy Bajo Impacto & 0583-2008-SETENA & $\begin{array}{l}\text { Actualización de la lista de proyectos de } \\
\text { muy bajo impacto ambiental }\end{array}$ \\
\hline Procedimiento ASA & 1287-2008-SETENA & $\begin{array}{l}\text { Procedimiento para el control y segui- } \\
\text { miento ambiental }\end{array}$ \\
\hline $\begin{array}{c}\text { Legitimación Presentación Planes } \\
\text { Reguladores }\end{array}$ & 2253-2008-SETENA & $\begin{array}{l}\text { Instrucción para ingreso de planes } \\
\text { reguladores }\end{array}$ \\
\hline $\begin{array}{l}\text { Proyectos de Muy Bajo Impacto Ampli- } \\
\text { ación Resolución }\end{array}$ & 2653-2008-SETENA & $\begin{array}{l}\text { Ampliación de lista de proyectos de muy } \\
\text { bajo impacto ambiental }\end{array}$ \\
\hline $\begin{array}{l}\text { Publicación de Estudios Impacto } \\
\text { Ambiental }\end{array}$ & Acuerdo Acta 0087-2008-SETENA & $\begin{array}{l}\text { Instructivo sobre publicación de Estu- } \\
\text { dios de Impacto Ambiental }\end{array}$ \\
\hline Publicaciones & Acuerdo Acta 0140-2008-SETENA & \\
\hline $\begin{array}{l}\text { Restablecimiento del Estudio de Diag- } \\
\text { nóstico Ambiental - (EDA) }\end{array}$ & 2286-2009-SETENA & Operativización de los EDA \\
\hline
\end{tabular}


Cuadro 1 (continuación)

Instrumentos y procedimientos técnicos de EIA, vigentes en Costa Rica.

\begin{tabular}{|c|c|c|}
\hline Nombre & Instrumento & Observación \\
\hline Guía Técnica E.D.A. & 2572-2009-SETENA & $\begin{array}{c}\text { Guía para la elaboración de Estudios de } \\
\text { Diagnóstico Ambiental }\end{array}$ \\
\hline Adendum Resolución & 730-2010-SETENA: & Asunto EDA \\
\hline Proceso Digitalización & 2342-2009-SETENA & Digitalización de información \\
\hline $\begin{array}{c}\text { Proceso Digitalización - Fecha Imple- } \\
\text { mentación }\end{array}$ & 2778-2009-SETENA & $\begin{array}{c}\text { Fecha de inicio de requisito de digita- } \\
\text { lización }\end{array}$ \\
\hline $\begin{array}{l}\text { Lista de chequeo y comunicado para re- } \\
\text { cepción de los Planes de Ordenamiento } \\
\text { Territorial y los Planes Maestros }\end{array}$ & 0213-2009-SETENA & $\begin{array}{l}\text { Requisitos de ingreso para planes } \\
\text { reguladores }\end{array}$ \\
\hline Vigencia de la Viabilidad Ambiental & 0147-2009-SETENA & Vigencia de la viabilidad \\
\hline $\begin{array}{l}\text { Rotulación Proyectos con Viabilidad A } \\
\text { y B1 }\end{array}$ & 1235-2009-SETENA & Rótulos ambientales de proyectos \\
\hline Bitácoras Ambientales & 2288-2011-SETENA & Lineamientos para bitácoras ambientales \\
\hline Renovación Consultores & 2912-2011-SETENA & $\begin{array}{l}\text { Lineamientos para renovación de } \\
\text { consultores }\end{array}$ \\
\hline $\begin{array}{c}\text { Instructivo Informe de Regencia } \\
\text { Ambiental }\end{array}$ & Acuerdo CP-036-2011-SETENA & $\begin{array}{l}\text { Lineamientos para Informes de "regen- } \\
\text { cia" ambiental }\end{array}$ \\
\hline $\begin{array}{l}\text { Procedimiento para el Establecimiento } \\
\text { de Medidas Compensatorias }\end{array}$ & Acuerdo CP-042-2011-SETENA & $\begin{array}{l}\text { Procedimiento para medidas compen- } \\
\text { satorias }\end{array}$ \\
\hline $\begin{array}{l}\text { Guía Ambiental para la Construcción } \\
\text { (Modifica Resolución 2827-2013-SETE- } \\
\text { NA en punto 17) }\end{array}$ & Resolución No. 479-2014-SETENA & $\begin{array}{c}\text { Modificación de la Guía Ambiental para } \\
\text { construcción }\end{array}$ \\
\hline
\end{tabular}

Fuente: Datos del autor y Página electrónica: www.setena.go.cr (junio del 2015).

EIA. Llama la atención que existe una relación inversamente proporcional, pues con proyectos pequeños y medianos, la exigencia es más alta; mientras que para proyectos de mayor tamaño, el grado de exigencia es más laxo.

\section{MARCO REGULATORIO}

El artículo 83 de la Ley Orgánica del Ambiente le da un marco operativo a la SETENA, al establecerla como "órgano de desconcentración máxima del Ministerio del Ambiente y Energía", y le señala que su "propósito fundamental será entre otros armonizar el impacto ambiental con los procesos productivos". Algo que la SETENA no ha definido con claridad qué significa y que, con frecuencia, se ha confundido con la idea de que la SETENA no puede decir que no!
La misma Ley del Ambiente, le da un mandato adicional, muy importante a la SETENA, y que a veces se olvida con frecuencia. En el artículo 86, referido al tema de Eficiencia, se señala que: "la Secretaría Técnica Nacional Ambiental deberá responder a las necesidades de eficiencia y eficacia en el análisis de las evaluaciones de impacto ambiental, de conformidad con las normas específicas, viables y funcionales para la conservación del ambiente orientada hacia el desarrollo sostenible".

Por su parte, la Procuraduría General de la República y la misma Sala Constitucional, han señalado que el proceso de EIA, es el principal instrumento que tiene el Estado, para tutelar el artículo 50 constitucional, particularmente en lo referente a garantizar un ambiente sano y ecológicamente equilibrado. Ello, en razón de que se trata de un 
instrumento preventivo y que el Estado puede decidir si dice sí o no a una actividad, obra o proyecto, antes de que el mismo pueda generar un eventual daño al ambiente.

Al respecto, la Sala Constitucional (Pronunciamiento 2001-13295) ha señalado lo siguiente:

“...que el Estado debe tomar todas las medidas técnicas posibles para asegurarse que la actividad que aprueba no causará daños al ambiente. Si bien el pronunciamiento del órgano estatal encargado es en sí un asunto técnico, el deber de pronunciarse y la exigencia de rigurosidad es un asunto jurídico. En términos generales, el deber estatal de garantizar el derecho a un ambiente sano no se reduce a satisfacer un simple requisito marginal dentro del proceso licitatorio. La Constitución no exige solo cumplir con ciertas formalidades, sino utilizar como se dijo todos los medios jurídica y fácticamente posibles para preservar el ambiente." (Ver en el mismo sentido la sentencia $\mathrm{N}^{\circ} 05906-99$ de las 16:15 hrs. de 28 de julio de 1999).

Y dentro de esa misma línea, sobre la SETENA, ha señalado lo siguiente:

"La preocupación ambiental del Ministerio del Ambiente y Energía se reduce prácticamente a los estudios de impacto ambiental, cuya aprobación está en manos de SETENA. Es decir, en SETENA recae todo el deber del Estado de garantizar un ambiente sano y ecológicamente equilibrado, como indica el artículo 50 constitucional. Ante tan importante responsabilidad, sería razonable esperar la fortaleza técnica y administrativa del órgano encargado."

Es en este sentido que, en materia ambiental, resulta especialmente grave toda conducta omisiva estatal que tenga como resultado un desmejoramiento de las condiciones del medio ambiente..."

Dentro de este contexto, el que el proceso de EIA no se realice de forma eficiente y efectiva tiene consecuencias muy serias, no solo limitadas al proyecto, obra o actividad, sino en general, al medio ambiente y en particular, al "modelo de desarrollo" que sigue el país, dado que el mismo pierde sustentabilidad ambiental.

\section{CONSECUENCIAS GENERALES}

Uno de los mayores problemas tiene que ver con los impactos acumulativos y sinergísticos que se producen. Los primeros tienen que ven con la suma combinada de impactos ambientales negativos significativos, que producen diferentes proyectos y que se van sumando.

Ejemplos de estos, son; las emisiones al aire, la producción de aguas pluviales por impermeabilización del terreno, la generación de residuos sólidos ordinarios y peligrosos, la contaminación del suelo y las aguas subterráneas, la afectación de biotopos (ecosistemas, flora y fauna), la modificación radical del paisaje, la potenciación antrópica de las amenazas naturales y efectos negativos en los patrones socioculturales, entre otros.

Por su parte, los efectos sinergísticos, abarcan la combinación de varios impactos negativos significativos y consecuencia en el medio ambiente. Un ejemplo de este tipo de impactos tiene que ver como la alteración de la calidad del aire como producto de los efectos ambientales combinados de diversas actividades o, también, la alteración integral del paisaje, por los cambios de uso del suelo producidos por el desarrollo urbano o las actividades agrícolas no planificadas.

En general, los efectos sinergísticos, se reflejan por un gradual, pero sistemático, deterioro de la calidad del ambiente y por el hecho concreto de que, los impactos negativos significativos de tipo residual, que producen los diversos proyectos, se transfieren al medio, precisamente porque no se logran controlar de forma eficiente dentro del área del proyecto. Ello, en razón de que la EIA no fue realizada de forma efectiva por los consultores ambientales y porque la SETENA no cumplió de manera eficiente con su labor.

\section{PROBLEMÁTICA DE LA GESTIÓN GEOLÓGICA AMBIENTAL}

Más específicamente, en el tema de la gestión ambiental que realiza un profesional en geología que ejerce como consultor ambiental, el problema 
de la falta de eficiencia en el proceso de EIA, se sustenta en una serie de problemas detectados. En lo que sigue, se citan los principales:

1. El geólogo consultor, con frecuencia incurre en errores importantes en los documentos que genera, particularmente en el denominado "Estudio Geológico del Terreno". Entre estos errores se incluyen:

a. No se cumple a cabalidad con el procedimiento técnico establecido por el Manual de EIA.

b. No se utiliza la escala apropiada (máximo 1:10.000), sino que se utilizan escalas mayores (generalmente 1:50.000 o más).

c. No se realiza una efectiva discusión sobre la condición de geoaptitud del terreno (ver Astorga \& Campos, 2001), por lo que el resultado del "estudio" no se concreta.

d. No se establece correctamente el valor de certidumbre de los datos fuente, por lo que en muchos casos, es incierta la precisión con que se llega las conclusiones técnicas.

e. Por lo general, al dar el visto bueno geológico del terreno, no se establecen de forma detallada las condiciones técnicas (incluyendo limitantes) bajo las cuales es posible dicho uso del suelo.

f. Los estudios hidrogeológicos realizados son generales y no cumplen el grado de precisión que solicita el Servicio Nacional de Aguas Subterráneas, Riego y Avenamiento (SENARA).

g. La evaluación de amenazas naturales, por lo general, es superficial y en muchos casos se basan en los datos de la Comisión de Emergencias cuyos mapas son parciales e incompletos, además de generales pues fueron elaborados a escala 1:50.000.

2. Dentro de la SETENA misma, se da el caso que el informe técnico geológico, no es revisado por un profesional en geología, sino por un profesional de otra disciplina, con lo que se contraviene la misma Ley del Colegio de Geólogos de Costa Rica. Además, la posibilidad de detectar errores en el estudio disminuye ostensiblemente.

Las consecuencias de todo esto, son muy serias:

1. Como la Ley orgánica del ambiente establece que en caso de daño ambiental, existe corresponsabilidad por parte del consultor ambiental, una deficiente revisión del documento técnico geológico realizado por el geólogo, puede llevar a que el mismo, en caso de presentarse un daño al ambiente, pueda ser imputado en una denuncia penal.

2. La responsabilidad del geólogo al dictaminar sobre la geoaptitud del terreno, en principio, "no expira", dado que a partir de su conclusión el terreno en cuestión es objeto del desarrollo planteado. De esta manera, si surgen problemas sobre la condición de geoaptitud del terreno aprobado, al primero que se va a buscar para que dé explicaciones es al geólogo responsable del estudio técnico.

\section{DISCUSIÓN GENERAL}

En todo proceso de Evaluación de Impacto Ambiental (EIA) existen dos componentes: a) los instrumentos y procedimientos técnicos y b) el operador técnico administrativo del proceso. En el caso de Costa Rica, el primer componente, está representado por el Reglamento General de EIA y el Manual Técnico (ver Tabla 1) y el segundo componente, por la SETENA y su organización técnica administrativa, así como el conjunto de consultores que tiene inscritos y registrados.

Al momento de examinar la eficiencia del proceso de EIA se hace necesario valorar los dos componentes.

En el caso del análisis aquí realizado, se ha observado que existen deficiencias patentes y reiteradas en el uso de los instrumentos técnicos. Deficiencias que se pueden achacar tanto a los consultores ambientales como a la misma SETENA.

En el caso de los instrumentos y procedimientos también se han detectado deficiencias e incluso inconsistencias, en particular entre los decretos ejecutivos vigentes y las resoluciones técnicas emitidas por la Comisión Plenaria de la SETENA (ver Cuadro 1).

A pesar de lo anterior, el peso principal del problema de eficiencia en el sistema de EIA, tiene que ver con los "operadores" y no tanto con los instrumentos. Se señala esto, en razón de que, como se ha visto aquí, el mayor problema 
de eficiencia tiene que ver con el incumplimiento de los procedimientos técnicos establecidos, tanto por los consultores ambientales como por la misma SETENA que es la responsable de la revisión.

Ante esto, la conclusión principal que se deriva es que, antes de cambiar instrumentos y procedimientos técnicos, se hace indispensable trabajar primero con los operadores, a fin de que se mejore de forma notable el proceso de EIA.

\section{POSIBLES SOLUCIONES}

Con el objetivo de mejorar la eficiencia del proceso de EIA, se sugieren cinco principales propuestas de solución:

1. Aprovechando la tecnología disponible, se considera de gran importancia que por medios digitales, todos los expedientes en trámite (y de ser posible, lo ya aprobados), puedan estar disponibles en una dirección electrónica oficial de la SETENA y que los mismos puedan ser accesados por el público. Se parte del principio lógico de que si un consultor sabe que sus documentos técnicos pueden estar bajo el escrutinio y revisión del público en general, va a tratar de cumplir con más precisión con los procedimientos establecidos.

2. También, se hace relevante que la SETENA cumpla la misma reglamentación sobre el registro y habilitación de consultores ambientales. En particular, sobre el requisito de conocimientos en EIA y de cursos de refrescamiento. Al respecto, se considera muy importante que la SETENA desarrolle una oficina especial que atienda este tema y la renovación de los consultores, cuyo costo de funcionamiento debería ser trasladado a los consultores ambientales. De igual manera, es fundamental contar con un Código de Ética del Gestión Ambiental y con mecanismos concretos de sanción por incumplimiento.

3. En lo referente a la SETENA misma es relevante que sus profesionales de planta, cumplan como mínimo con los requisitos que se establece a los consultores ambientales y que, por lo tanto, puedan ser objeto de evaluación periódica. Además, es relevante que las evaluaciones de expedientes se realicen de forma interdisciplinaria y no por un solo profesional. También es clave que de todos los procesos de análisis se disponga de formularios, por medio de los cuales, se registren los criterios de ponderación utilizados. Señalando además, los profesionales que participaron en la evaluación. Estos formularios deben ser parte del expediente y estar disponibles al público de forma digital.

4. Es de gran importancia que se fortalezca el proceso de Control y Seguimiento Ambiental de proyectos, obras y actividades en operación. Es relevante que se implementen los instrumentos que da la legislación tales como: las inspecciones y auditorías ambientales de cumplimiento (externas) y la calificación ambiental, entre otras. Además, resulta de gran importancia que una copia de los informes ambientales de "regencia”, puedan ser entregados a la Municipalidad del cantón donde se localiza el proyecto a fin de que la misma pueda ejercer también un proceso de control. Dichos informes también deberían ser parte del expediente digital y por tanto, poder ser accesados por el público. Además, la SETENA debería cobrar por la revisión de dichos informes a fin de que pueda tener más equipo profesional en este proceso.

5. En lo referente a los instrumentos técnicos también es relevante que se realice una revisión y una modernización, pero dentro de la línea de que sean mejorados, no necesariamente sustituidos. Se hace clave armonizar el conjunto de decretos ejecutivos del Manual de EIA con respecto a las resoluciones emitidas por la Comisión Plenaria de la SETENA. En particular a la luz de lo que señala la Ley 8220 y sus modificaciones, así como su reglamento. Al respecto, no se debe olvidar la importancia de las modificaciones y mejoras de los instrumentos de EIA, debe hacerse de forma participativa y con criterio técnico. Razón por la cual, el Reglamento General de EIA, creo una Comisión Mixta asesora de la SETENA, que tiene su propio reglamento emitido por decreto ejecutivo y que, debería ser la encargada de discutir y modernizar la "caja de herramientas" técnicas que regulan el proceso de EIA de Costa Rica. 


\section{REFERENCIAS BIBLIOGRÁFICAS}

CANTER, L. W. (1997): Manual de Evaluación de Impacto Ambiental. Técnicas para la elaboración de los estudios de impacto ambiental.- 841 págs. McGraw Hill, New York.

ASTORGA, A. \& CAMPOS, L., (2001): El cartografiado de geoaptitud de terrenos.- Rev. Geol. Amer. Central, 24: 103-110.

MINAE (2004): Manual de Instrumentos Técnicos para el Proceso de Evaluación de Impacto Ambiental (Manual de EIA) -PARTE I-. Instructivo de llenado del Formulario D2 y Código de Buenas Prácticas Ambientales. No. 32079.- La Gaceta, 217: 117-147.

MINAE, (2005a): Manual de Instrumentos Técnicos para el Proceso de Evaluación de Impacto Ambiental (Manual de EIA) -PARTE II-. Instructivo de llenado del Formulario D1. No. 32712.- La Gaceta, 223: 149-238.

MINAE (2005b): Creación de la Comisión Nacional Asesora Técnica Mixta de la Secretaría Técnica Nacional Ambiental (SETENA) [Decreto 32631].- La Gaceta, 197: 31-35.

MINAE, (2006a): Manual de Instrumentos Técnicos para el proceso de Evaluación de Impacto Ambiental (Manual de EIA) -PARTE IV-. Guía para la elaboración de instrumentos de EIA y procedimiento de valoración de impactos ambientales. [Decreto 32966].- La Gaceta, 85: 309-344.
MINAE, (2006b): Manual de Instrumentos Técnicos para el proceso de Evaluación de Impacto Ambiental (Manual de EIA) PARTE III-. Decreto 32967. Procedimiento para introducir la variable ambiental en los planes reguladores y planificación de uso del suelo.- La Gaceta, 85: 239-307.

MINAE, (2007). Manual de Instrumentos Técnicos para el Proceso de Evaluación de Impacto Ambiental (Manual de EIA) -PARTE V-. Procedimiento técnico y ambiental para los movimientos de tierras [Decreto 33959].- La Gaceta, 244: 2-3.

MINAE, (2008a): Reglamento para la elaboración, revisión y oficialización de las Guías Ambientales de buenas prácticas productivas y desempeño ecoeficiente. No. 34522.La Gaceta, 115: 20-33.

MINAE, (2008b): Reglamento de fijación de tarifas de servicios brindados por la Secretaría Técnica Nacional Ambiental [Decreto 34536] .- La Gaceta, 105: 12-16.

MINAET, (2011): Reglamento de Organización de la Estructura Interna de Funcionamiento de la Secretaría Técnica Nacional Ambiental - SETENA [Decreto 36815].- La Gaceta, 213: 2-9.

MINAE - SALUD - MOPT - MAG - MEIC, (2004): Reglamento General sobre los procedimientos de Evaluación de Impacto Ambiental (EIA). [Decreto 31.849].- La Gaceta, 125: 37-116. 
